\title{
A dual-slice $k$-t approach for highly accelerated flow MRI
}

\author{
Daniel Giese ${ }^{1,2^{*}}$, Tobias Schaeffter ${ }^{2}$, Sebastian Kozerke ${ }^{1,2}$ \\ From 15th Annual SCMR Scientific Sessions \\ Orlando, FL, USA. 2-5 February 2012
}

\begin{abstract}
Summary
We propose the combination of $k-t$ undersampling with a dual slice excitation approach. The dual slice excitation and reconstruction principle is incorporated into the $k-t$ SENSE reconstruction framework, allowing a doubling of the net acceleration factor when acquiring two slices as compared to acquiring two separate undersampled slices.
\end{abstract}

\section{Background}

Phase contrast MRI has limited practical value in clinical applications due to its inherently long scan times [Kilner,JCMR'07]. Parallel imaging[Pruessmann, MRM'01] and spatio-temporal constrained reconstruction techniques[Tsao,MRM'03] have been shown beneficial[Baltes,MRM'05]. Dual slice approaches have shown advantages as compared to standard SENSE when multiple and sufficiently separated slices are acquired[Breuer, MRM'10]. We present a combination of dual slice excitation with undersampled flow imaging by modifying the $k$ - $t$ SENSE reconstruction framework to include sensitivity information in through-slice direction.

\section{Methods}

The dual slice excitation was performed using a cosine modulated sinc pulse. A FOV/2 shift of one slice in phase encoding direction was achieved by alternating the phase of the RF pulse along the phase encoding direction. Dual slice $k-t$ undersampled data was acquired in a healthy volunteer in a single breathhold using a 32 channel coil array. The transversal slices were separated by $120 \mathrm{~cm}$, one was placed at the level of the pulmonary artery, the other one at the level of the liver. Slices were flow encoded with a velocity encoding of $200 \mathrm{~cm} / \mathrm{s}$. Prior

${ }^{1}$ Institute for Biomedical Engineering, University and ETH Zurich, Zurich, Switzerland

Full list of author information is available at the end of the article to reconstruction, the training data was SENSEunfolded. The $k-t$ unfolding was modified and expanded by incorporating the dimension of the two acquired slices resulting in a single inversion process with doubled matrix size as compared to single slice $k-t$ reconstruction (Figure 1). A nominal acceleration factor of $\mathrm{R}=5$ was chosen with 11 training profiles (net acceleration factor of 3.6 per slice totaling 7.2 for the dual slice acquisition). For comparison, reference fully sampled as well as undersampled acquisitions of each seperate slice were acquired. Root mean squared errors (RMSE) of the flow profiles (in $\mathrm{ml} / \mathrm{s}$ and \% of the maximum flow) were calculated for dual slice $k-t$ SENSE and single slice $k-t$ SENSE.

\section{Results}

Figure 2 shows $k-t$ dual-slice unfolded magnitude and phase images. Temporal behavior and flow quantification are demonstrated by flow curves of the ascending aorta in the upper slice and the descending aorta in both slices. Reference flow curves along with single slice $k-t$ SENSE and the proposed dual slice $k-t$ approach are plotted showing similar temporal behaviors. RMSE with respect to fully sampled data confirm the agreement between flow curves.

\section{Conclusions}

We have presented a reconstruction framework allowing reconstruction of two simultaneously acquired spatiotemporally undersampled phase-contrast slices. Initial results show good agreement between reference, fully sampled, single slice undersampled $k-t$ SENSE and the proposed dual slice accelerated acquisitions. Slight variations between all three images are likely due to the differences in sequentially acquired data. Future work will include the adaptation into other spatio-temporal constrained reconstruction techniques $(k-t$ PCA[Pedersen, 

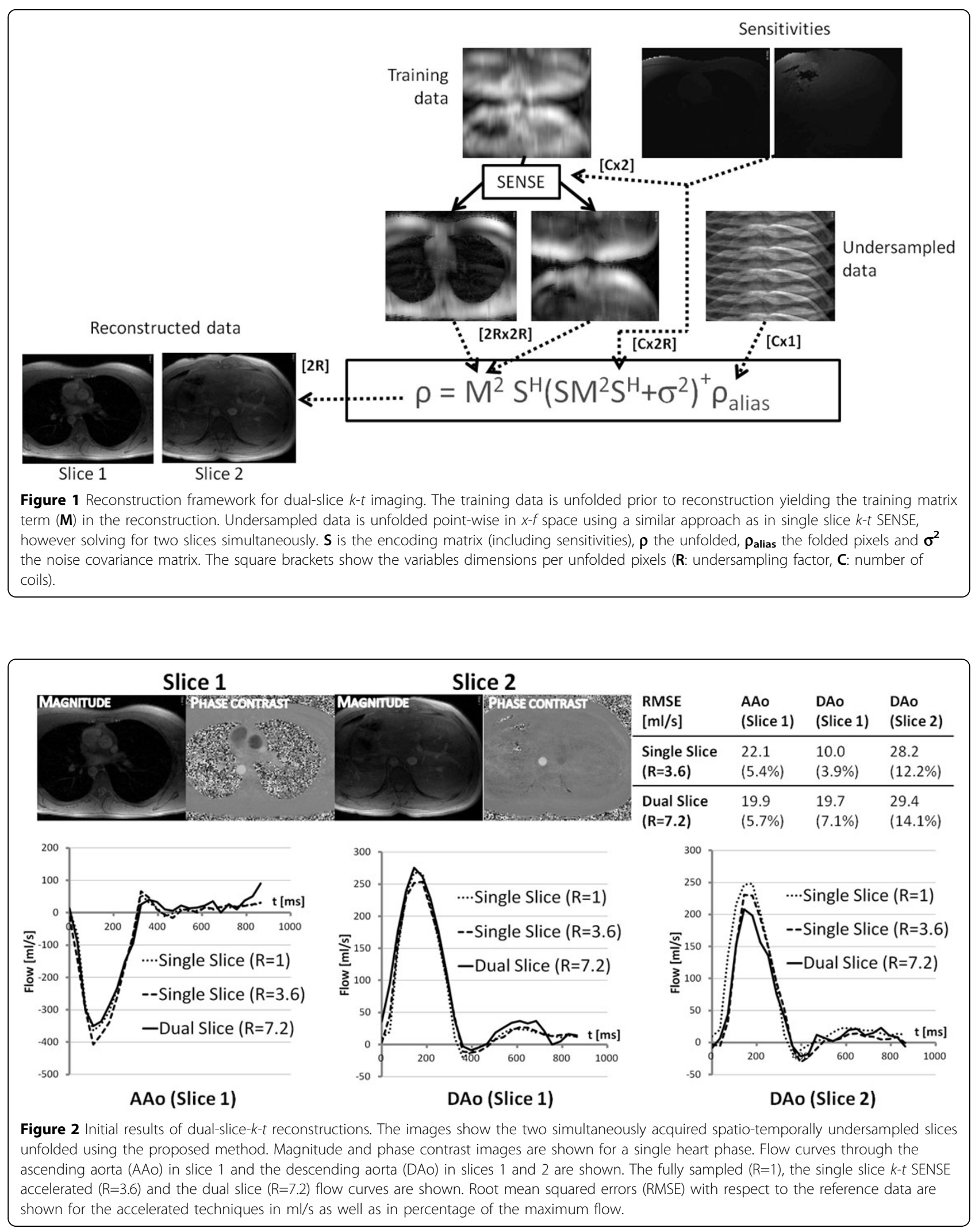
MRM'09]) allowing for higher net acceleration factors as well as validation in volunteers and patients.

\section{Author details}

'Institute for Biomedical Engineering, University and ETH Zurich, Zurich,

Switzerland. ${ }^{2}$ Division of Imaging Sciences and Biomedical Engineering,

King's College London, London, UK.

Published: 1 February 2012

doi:10.1186/1532-429X-14-S1-W47

Cite this article as: Giese et al: A dual-slice $k$ - $t$ approach for highly

accelerated flow MRI. Journal of Cardiovascular Magnetic Resonance 2012

14(Suppl 1):W47.

Submit your next manuscript to BioMed Central and take full advantage of:

- Convenient online submission

- Thorough peer review

- No space constraints or color figure charges

- Immediate publication on acceptance

- Inclusion in PubMed, CAS, Scopus and Google Scholar

- Research which is freely available for redistribution

Submit your manuscript at www.biomedcentral.com/submit 